Corrigendum

\title{
Corrigendum to "Subchronic Infection of Porphyromonas gingivalis and Tannerella forsythia Stimulates an Immune Response but Not Arthritis in Experimental Murine Model"
}

\author{
Jorday Hernández-Aguas, ${ }^{1}$ José Luis Montiel-Hernández, ${ }^{2}$ \\ Myriam A. De La Garza-Ramos $\mathbb{D}^{1},{ }^{1}$ Rosa Velia Ruiz-Ramos, ${ }^{1}$ Erandi Escamilla García $\mathbb{D}^{\mathbb{D}}{ }^{1}$ \\ Mario Alberto Guzmán-García, ${ }^{3}$ Esperanza Raquel Ayón-Haro, \\ and Mario Alberto Garza-Elizondo ${ }^{1}$ \\ ${ }^{1}$ Universidad Autónoma de Nuevo Leon, Centro de Investigación y Desarrollo en Ciencias de la Salud (CIDICS), Facultad de \\ Odontología, Calle Dr. Eduardo Aguirre Pequeño s/n, Colonia Mitras Centro, 64460 Monterrey, NL, Mexico \\ ${ }^{2}$ Universidad Autónoma del Estado de Morelos, Facultad de Farmacia, Avenida Universidad 1001, 62209 Cuernavaca, \\ MOR, Mexico \\ ${ }^{3}$ Universidad Autónoma de Nuevo Leon, Facultad de Medicina Veterinaria y Zootecnia, Centro de Investigación y Desarrollo en \\ Ciencias de la Salud (CIDICS), Calle Francisco Villa s/n Colonia Ex-Hacienda el Canada, 66050 Escobedo, NL, Mexico \\ ${ }^{4}$ Universidad de San Martín de Porres, Laboratorio de Investigación en Biología Oral y Molecular, Ciudad Universitaria, Jr. Las \\ Calandrias $N^{\circ}$ 151-291, Santa Anita, Lima 15009, Peru
}

Correspondence should be addressed to Myriam A. De La Garza-Ramos; myriam.garzarm@uanl.edu.mx

Received 21 November 2017; Accepted 26 August 2018; Published 1 October 2018

Copyright ( $\odot 2018$ Jorday Hernández-Aguas et al. This is an open access article distributed under the Creative Commons Attribution License, which permits unrestricted use, distribution, and reproduction in any medium, provided the original work is properly cited.

In the article titled "Subchronic Infection of Porphyromonas gingivalis and Tannerella forsythia Stimulates an Immune Response but Not Arthritis in Experimental Murine Model" [1], there was an error in Table 1 where "Freud's adjuvant" should be changed to "Freund's adjuvant." The formatting of the table is also being corrected for clarity. The correct table is as follows.

TABLE 1: Inflammatory response of the hind limbs of Balb/c mice.

\begin{tabular}{|c|c|c|}
\hline Groups & Hind limb swelling $(\mathrm{mm})$ & Hind limb thickness $(\mathrm{mm})$ \\
\hline $\begin{array}{l}\text { Group } 1 \\
\text { Sham } \\
\text { Sham } \\
\text { Sham }\end{array}$ & $\begin{array}{l}\text { R: } 1.4-\mathrm{L}: 1.4 \\
\text { R: } 1.4-\mathrm{L}: 1.4 \\
\text { R: } 1.4-\mathrm{L}: 1.4 \\
\end{array}$ & $\begin{array}{l}\text { R: } 2-\mathrm{L}: 2 \\
\text { R: } 2-\mathrm{L}: 2 \\
\mathrm{R}: 2-\mathrm{L}: 2\end{array}$ \\
\hline $\begin{array}{l}\text { Group } 2 \\
\text { Sham }+P . \text { gingivalis plus } T . \text { forsythia } \\
\text { Sham }+P . \text { gingivalis plus } T . \text { forsythia } \\
\text { Sham }+P . \text { gingivalis plus } T \text {. forsythia }\end{array}$ & $\begin{array}{l}\text { R: } 1.4-\mathrm{L}: 1.4 \\
\text { R: } 1.4-\mathrm{L}: 1.4 \\
\text { R: } 1.4-\mathrm{L}: 1.4\end{array}$ & $\begin{array}{l}\text { R: } 2-\mathrm{L}: 2 \\
\mathrm{R}: 2-\mathrm{L}: 2 \\
\mathrm{R}: 2-\mathrm{L}: 2\end{array}$ \\
\hline $\begin{array}{l}\text { Group } 3 \\
P g / T f \text { plus Freund's adjuvant } \\
P g / T f \text { plus Freund's adjuvant } \\
P g / T f \text { plus Freund's adjuvant }\end{array}$ & $\begin{array}{l}\text { R: } 1.4-\mathrm{L}: 1.4 \\
\text { R: } 1.4-\mathrm{L}: 1.4 \\
\text { R: } 1.4-\mathrm{L}: 1.4\end{array}$ & $\begin{array}{l}\text { R: } 2.5-\mathrm{L}: 2.5 \\
\mathrm{R}: 2.5-\mathrm{L}: 2.5 \\
\mathrm{R}: 2.5-\mathrm{L}: 2.5\end{array}$ \\
\hline $\begin{array}{l}\text { Group } 4 \\
\text { Freund's adjuvant } \\
\text { Freund's adjuvant } \\
\text { Freund's adjuvant }\end{array}$ & $\begin{array}{l}\text { R: } 1.4-\mathrm{L}: 1.4 \\
\text { R: } 1.5-\mathrm{L}: 1.5 \\
\text { R: } 1.4-\mathrm{L}: 1.4\end{array}$ & $\begin{array}{l}\text { R: } 2.5-\mathrm{L}: 2.5 \\
\mathrm{R}: 2.5-\mathrm{L}: 2.5 \\
\mathrm{R}: 2.5-\mathrm{L}: 2.5\end{array}$ \\
\hline
\end{tabular}

$\mathrm{R}=$ right; $\mathrm{L}=$ left $P g / T f=P$. gingivalis plus $T$. forsythia; sham = placebo. 


\section{References}

[1] J. Hernández-Aguas, J. L. Montiel-Hernández, M. A. De La GarzaRamos et al., "Subchronic Infection of Porphyromonas gingivalis and Tannerella forsythia Stimulates an Immune Response but Not Arthritis in Experimental Murine Model," International Journal of Dentistry, vol. 2017, Article ID 2052938, 7 pages, 2017. 


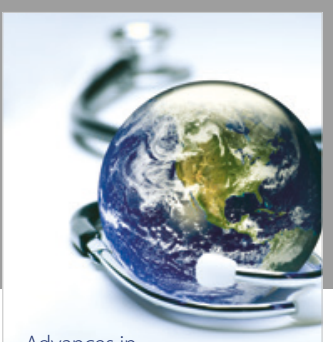

Advances in
Public Health

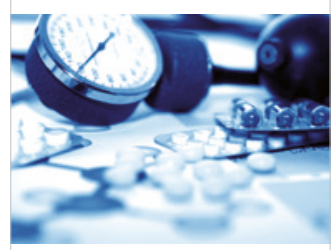

Case Reports in

Medicine

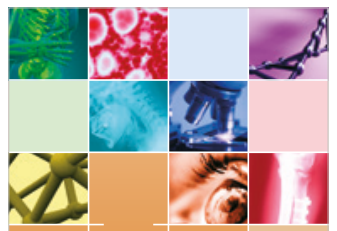

niernational Journal of

Biomaterials
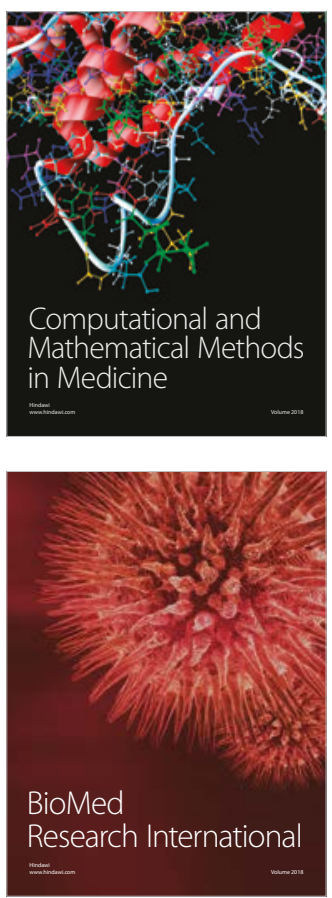

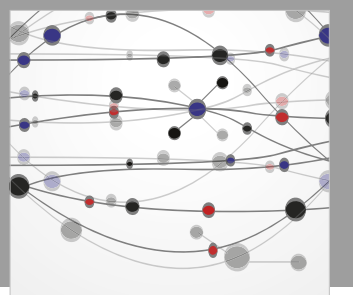

The Scientific World Journal Dentistry

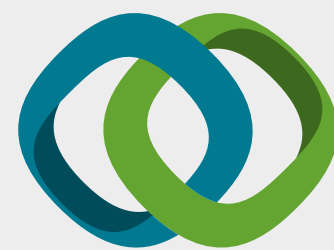

Hindawi

Submit your manuscripts at

www.hindawi.com
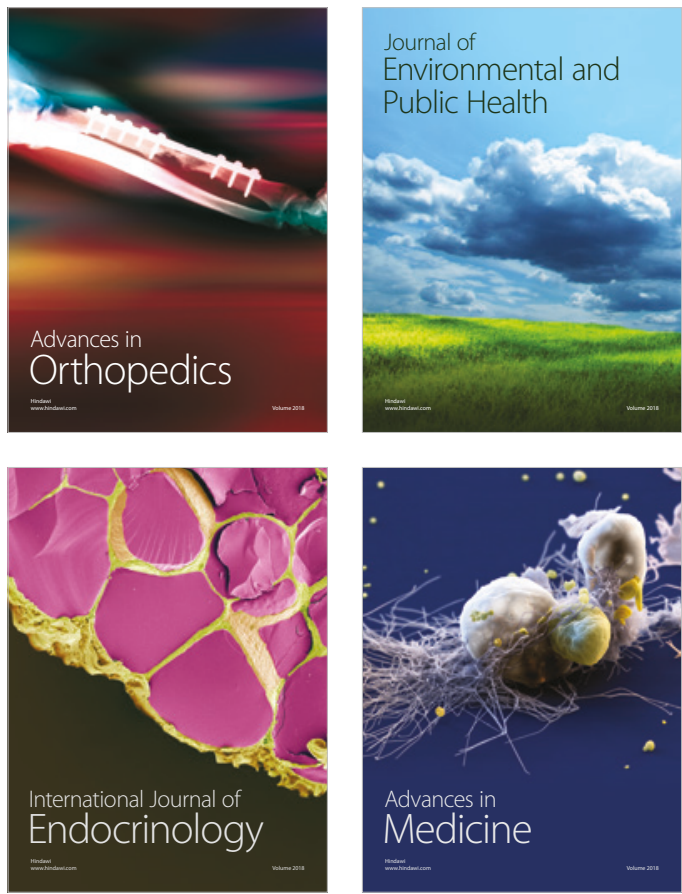
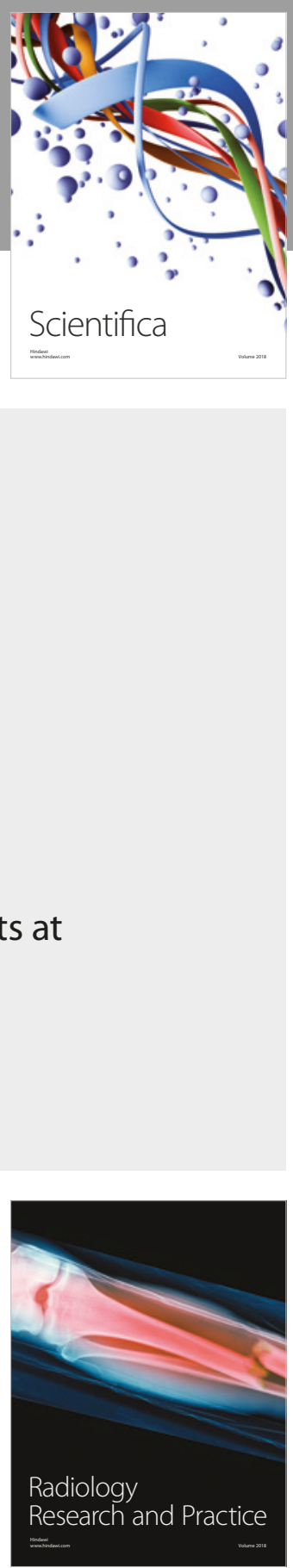

Scientifica

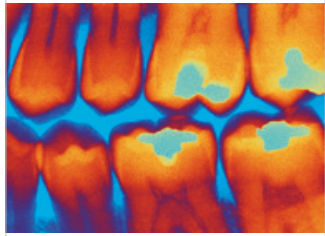

Case Reports in

Dentistry
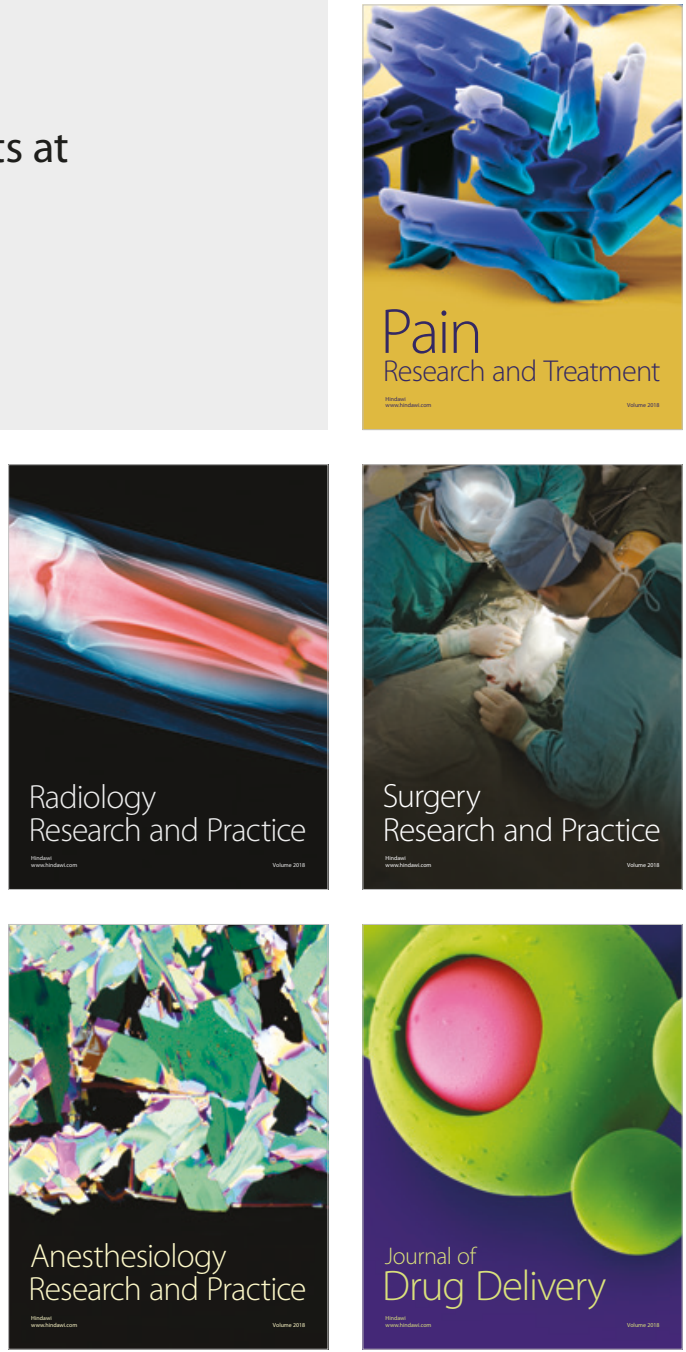